\title{
editorials
}

\section{A new look for the journal}

THE JOURNAL OF THE AMERICAN OSTEOPATHIC ASSOCIATION is the official scientific publication of the American Osteopathic Association. JAOA has a dual function of documenting osteopathic contributions in clinical/scientific research and providing clinical teaching for its physician readers. However, the increased diversity of education, training, interests, and clinical practice of osteopathic physicians has made it more difficult for JAOA to maintain a wide appeal to its readership.

For the past several months, the editorial staff of JAOA has reviewed the results of readership surveys, conducted sample interviews, and discussed with the editorial board several ideas for better serving the profession. The January issue reflects the decision to sectionalize JAOA to provide a wider range of articles, from academic/research-oriented pieces to those aimed at the clinical practitioner. Sections will include: (1) Original Contributions-a monthly section of articles focusing on original clinical research and significant basic research; (2) Case Reports-a monthly section featuring both documental and teaching case reports; (3) Clinical Practice-articles highlighting techniques that have applications for both general practitioners and specialists; (4) Medical Education-documentation of the work of our educators that focus on medical students and the colleges; (5) Letters to the Editor-substantive comments on previous articles; (6) Brief Reports-solid but concise documentation of clinical information; and (7) Special Communications-lectures, informed commentary on health care, or communication from AOA officers.

JAOA is your journal. Your contributions and feedback to the editorial staff will enable them to respond to your needs. Let us know what you think.

THOMAS W. ALLEN, D.O., FACOI

\section{The somatic component to visceral disease}

The viscerosomatic interrelationship has always been a basic tenet of the osteopathic concept. The principle of somatic manifestation of visceral disease is fundamental to the practice of osteopathic medicine. Many investigators have examined this "reflex" phenomenon in a wide range of visceral disorders in laboratory animals and in humans. Another important controlled clinical trial that contributes to the scientific basis for this osteopathic tenet is featured in this issue of JAOA.

The study, "Somatic manifestations in renal disease," which begins on page 22/61, was conducted by Johnston, Kelso, Hollandsworth, and Karrat. These researchers' data support the presence of somatic motor and vasomotor changes existing as reflex components of renal disease. The authors offer several possible mechanisms for the viscerosomatic reflex, all suggesting nervous control of visceral motor tone.

Osteopathic physicians have long recognized the presence of musculoskeletal findings in patients with visceral disease. As the authors note, the visceral reflex disturbance on somatic motor function is often self maintained even after the visceral source is alleviated or surgically removed. They note that the goal of the manipulative approach is to interrupt the disturbed cycle of spinal reflexes in the facilitated pathways.

THOMAS W. ALLEN, D.O., FACOI

\section{Cholesterol and coronary heart disease}

The relationship between serum cholesterol and coronary heart disease has been the focus of extensive investigation for the past 25 years. Results from a variety of epidemiologic and clinical investigations have resulted in the consensus that lowering serum cholesterol levels significantly reduces the incidence of myocardial infarction in high-risk adult men. The most recent investigation by the Lipid Research Clinics Coronary Primary Prevention Trial (LRC-CPPT) provides conclusive evidence of this benefit. The multicenter, randomized, double-blind study of 3,806 men with serum cholesterol levels $\geqslant 265 \mathrm{mg}$./dl. was conducted from 1976 through 1983 . The trial showed that a reduction in serum cholesterol through dietary modifica- 